\title{
An energy-efficient readout circuit for resonant sensors based on ring-down measurement
}

\author{
Z. Zeng, ${ }^{1}$ M. A. P. Pertijs, ${ }^{2}$ and D. M. Karabacak ${ }^{1}$ \\ ${ }^{1}$ Holst Centre/imec, High Tech Campus 31, Eindhoven, The Netherlands \\ ${ }^{2}$ Electronic Instrumentation Laboratory/DIMES, Delft University of Technology, Delft, The Netherlands
}

(Received 4 January 2013; accepted 1 February 2013; published online 25 February 2013)

\begin{abstract}
This paper presents an energy-efficient readout circuit for resonant sensors that operates based on a transient measurement method. The resonant sensor is driven at a frequency close to its resonance frequency by an excitation source that can be intermittently disconnected, causing the sensor to oscillate at its resonance frequency with exponentially decaying amplitude. By counting the zero crossings of this ring-down response, the interface circuit can detect the resonance frequency. In contrast with oscillator-based readout, the presented readout circuit is readily able to detect quality factor $(Q)$ of the resonator from the envelope of the ring-down response, and can be used even in the presence of large parasitic capacitors. A prototype of the readout circuit has been integrated in $0.35 \mu \mathrm{m}$ CMOS technology, and consumes only $36 \mu \mathrm{A}$ from a $3.3 \mathrm{~V}$ supply during a measurement time of $2 \mathrm{~ms}$. The resonance frequency and quality factor of a micro-machined $\mathrm{SiN}$ resonator obtained using this prototype are in good agreement with results obtained using impedance analysis. Furthermore, a clear transient response is observed to ethanol flow using the presented readout, demonstrating the use of this technique in sensing applications. (C) 2013 American Institute of Physics. [http://dx.doi.org/10.1063/1.4792396]
\end{abstract}

\section{INTRODUCTION}

Micro/nano-mechanical resonators are considered to be well suited candidates for many sensing applications, especially for integrated systems, owing to their inherent advantages of low-power consumption, integratability, arrayability, and high sensitivity. Among the emerging applications for micro/nano-mechanical resonators are detection of biomolecules and chemical compounds. ${ }^{1}$ For instance, polymer-coated high-aspect (length to thickness) ratio clamped-clamped beam resonators with integrated piezoelectric transducers have been demonstrated to be highly responsive to low concentrations of volatile organic compounds. ${ }^{2}$

Clearly, there exists a need to develop an energy-efficient approach to readout micro/nano-mechanical resonators to fulfill their promise as integrated sensing elements. Ideally, the readout circuit needs to be capable of tracking miniscule changes in modal properties of the resonator while consuming minimum energy per measurement. Here, it is important to note that not only does the modal frequency provide a measure of inertial and tensional effects on the resonator, ${ }^{2}$ the quality factor of the resonance mode can also yield valuable information, especially to determine damping and energy loss effects, for example, due to either surface binding or pressure changes. ${ }^{3}$ Therefore, it is desirable that the readout approach is also capable of detecting the quality factor.

Although the sensitivity of resonators is highly favored by scaling into the micro/nanometer range, and piezoelectric transduction is considered to be compatible with such scaling, ${ }^{4,5}$ signal levels rapidly diminish as a result of scaling, especially relative to several sources of parasitic effects. ${ }^{6}$ This is an important challenge still to be addressed from the readout circuit architecture side.
Here, we explore the potential of adapting ring-down measurement ${ }^{7,8}$ to readout micro-mechanical resonators, compared with existing alternatives, and present in detail a low-power integrated readout circuit based on ring-down measurement. ${ }^{9}$

The paper is organized as follows: Section II presents an electrical model of the resonators and Sec. III discusses the more commonly employed techniques for resonant detection. Section IV details the operating principle of the proposed ring-down-based readout, and its circuit implementation is described in Sec. V. Section VI shows experimental results obtained using a prototype chip, and conclusions are drawn in Sec. VII.

\section{DEVICE CHARACTERISTICS}

The micro-machined resonant sensor used here is based on a silicon nitride ( $\mathrm{SiN})$ clamped-clamped beam with integrated piezoelectric transducer, as shown in Fig. 1(a). The devices, fabricated using standard microfabrication techniques, ${ }^{2}$ have typical dimensions of $100 \mu \mathrm{m}$ to $1000 \mu \mathrm{m}$ in length, $20 \mu \mathrm{m}$ to $100 \mu \mathrm{m}$ in width, and $500 \mathrm{~nm}$ SiN thickness. The transducer layer is formed by aluminum nitride (AIN) sandwiched between two metal electrodes, covering a quarter of the suspended region.

Within a narrow bandwidth of the modal frequency, the admittance of a resonator can be modeled as a $R L C$ tank (mechanical resistance $R_{m}$, capacitance $C_{m}$, and inductance $L_{m}$ ) in parallel with a parasitic shunt capacitor $C_{p}$ which represents the capacitance of transducer patch, ${ }^{10}$ as shown in Fig. 1(b). The real and imaginary components of admittance of the resonator (i.e., its conductance and susceptance), measured 
(a)

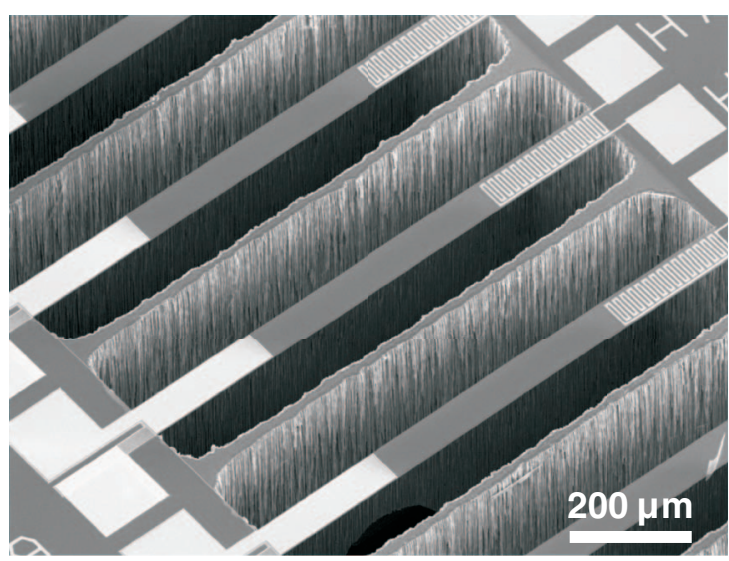

(b)

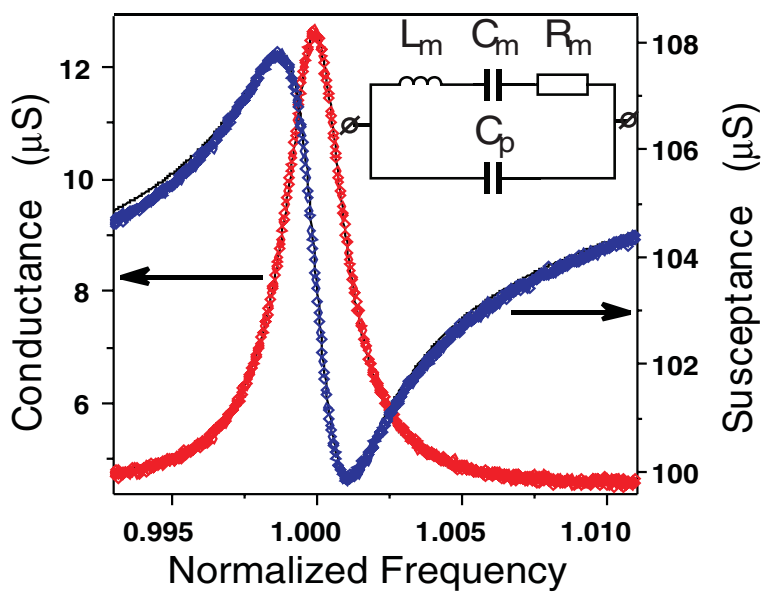

FIG. 1. (a) Scanning electron microscope image of resonator beams. (b) Measured (markers) and modeled (lines) electrical conductance and susceptance of the resonator as a function of frequency (normalized), with inset showing the equivalent circuit used.

using an impedance analyzer (Agilent 4249A), can be well fitted using this electrical model, as shown in Fig. 1(b). From this fit, the resonance frequency $\left(f_{\text {res }}\right)$ and the quality factor $(Q)$ of a specific resonance mode can be determined as

$$
\begin{gathered}
f_{\text {res }}=\frac{1}{2 \pi \sqrt{L_{m} C_{m}}}, \\
Q=\frac{1}{R_{m}} \sqrt{\frac{L_{m}}{C_{m}}} .
\end{gathered}
$$

For the resonators described above, typical values of $f_{\text {res }}$ can range from $0.1 \mathrm{MHz}$ to $2 \mathrm{MHz}$ with quality factor $Q$ in the range of 50 to 600 in air, depending on dimensions and vibration modes of operation.

\section{READOUT APPROACHES}

There exist three main established approaches to measure the modal frequency and quality factor of resonators: impedance analysis, oscillator-based readout, and ring-down measurement. Here, we discuss and compare these approaches within the context of piezoelectric MEMS resonators.

\section{A. Impedance analysis}

Impedance analysis is the most commonly used technique for characterization of resonant sensors using bench-top equipment. ${ }^{11,12}$ The electrical impedance or admittance of the resonant sensor is measured over a narrow range of frequency around the modal frequency, recording both amplitude and phase response. The obtained complex impedance or conductance is fitted with the transfer function to extract the equivalent circuit model and also, the resonance frequency and quality factor, as described in Sec. II. While being very flexible in its operating range and providing accurate measurements of the spectral behaviors of the resonators, impedance analysis tends to be a relatively time-consuming and computationally intensive procedure, and is thus not readily applicable to lowpower sensor applications.

\section{B. Oscillator-based readout}

Alternatively, a resonant element can be connected in a positive feedback loop with a sustaining amplifier to form a circuit that oscillates at a frequency determined by the mechanical resonator. The sustaining amplifier ensures that the motional current from the transducer is stable by providing a controlled actuation voltage that is matched in phase with the detected current. ${ }^{11,13,14}$ The resulting oscillation frequency is, therefore, very close to the resonance frequency, and can then be measured by frequency counting. The oscillator-based readout scheme has been previously demonstrated to be very accurate in tracking the resonance frequency of the aforementioned resonators. ${ }^{15,16}$ However, several technical limitations remain for this approach. It requires the sustaining amplifier to have a well-defined phase shift, which comes at the cost of power consumption, typically around $1 \mathrm{~mW}$ for the resonators discussed here. Moreover, with large electrical parasitics, the phase shift of the resonator at resonance becomes small and poorly defined, particularly in $\mu \mathrm{m}$ scale transducers, making it difficult for the sustaining amplifier to provide sufficient phase shift to sustain oscillation without additional efforts of parasitic capacitance cancellation. ${ }^{15,16}$ Furthermore, oscillator-based readout cannot readily provide information about the quality factor, which can be used to quantify damping effects on the surface of the resonator or the functionalization layer in some sensing applications.

\section{Ring-down measurement}

A ring-down measurement consists of two phases. ${ }^{7,8}$ During a first excitation phase, the resonator is driven by an excitation source with a driving frequency within close vicinity of the desired modal frequency. During a second phase, the source is disconnected, and the subsequent transient response of the resonator is captured. The frequency of this ring-down signal ideally equals the resonance frequency. Its amplitude, which decays as the resonator losses energy due to the mechanical damping factor, contains information about the quality factor. To extract the resonance frequency and quality factor, curve-fitting can be applied to the captured ring-down signal. ${ }^{8}$ A less complex approach is to use 


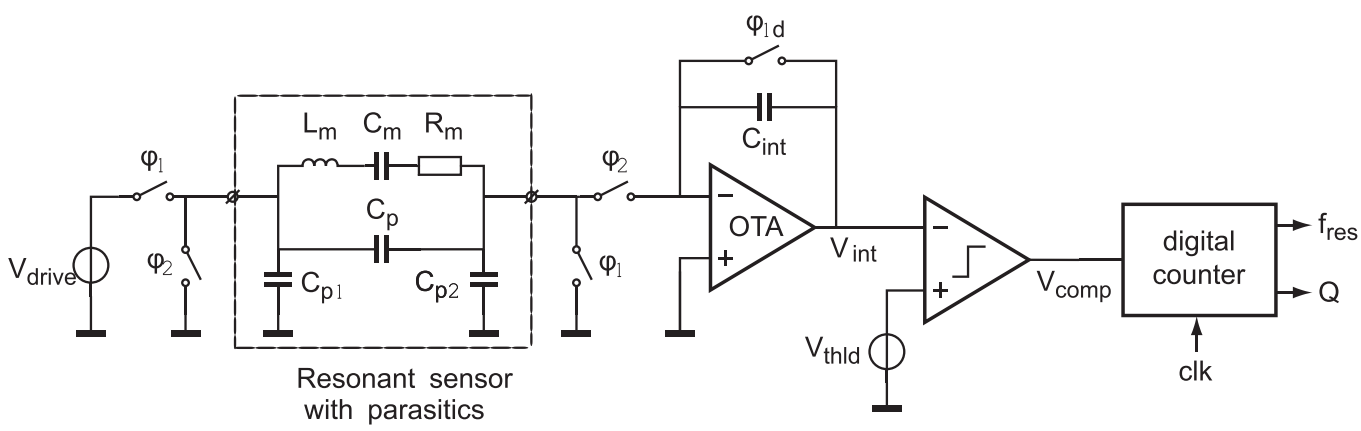

FIG. 2. Proposed architecture of the interface circuit, consisting of a switched excitation source, a resonant sensor with parasitics, a switched-capacitor currentto-voltage converter (using an operational trans-conductance amplifier), a comparator, and a digital counter.

frequency counting technique: the resonance frequency can be calculated by counting the zero-crossings of the ring-down signal, ${ }^{17}$ while the quality factor can be estimated by counting threshold-crossings of the ring-down signal at non-zero threshold levels. ${ }^{18}$

Therefore, compared to impedance analysis, the ringdown measurement can extract the resonance frequency and quality factor by using computationally simpler counting techniques. Compared to oscillator-based readout, the ringdown technique can be designed less sensitive to parasitics, can readily extract quality factor, and also operate with significantly lower power consumption. However, one disadvantage of this technique is that the resonance frequency needs to be approximately known a priori for efficient excitation of the resonator, although this limitation can be overcome by searching for the frequency with maximum ring-down signal within a narrow frequency band, and hence finding the resonance mode.

Some approaches based on the ring-down measurement have been previously reported in literature, using printed circuit board (PCB) level implementations, and mainly focusing on quartz crystal resonators. ${ }^{17,18}$ However, its application to micro-machined resonators, and its potential advantages in terms of energy-efficient readout of resonators are largely unexplored. Furthermore, an integrated implementation of the technique has not been reported in literature, to the best of the authors' knowledge.

\section{OPERATING PRINCIPLE OF RING-DOWN DETECTION}

The proposed readout architecture, shown in Fig. 2, consists of a front-end circuit (containing a switched excitation source, a resonant sensor, and a switched-capacitor current-to-voltage converter), a comparator, and a digital counter. During a first phase $\phi_{1}$, the resonator is driven by the excitation source. During a second phase $\phi_{2}$, the currentto-voltage converter records the resultant ring-down current to characterize the series resonance.

\section{A. Front-end operation}

During the excitation phase $\phi_{1}$, the driving source provides a voltage $V_{\text {drive }}$ (Fig. 3) at a frequency close to the reso- nance frequency of the resonant sensor, thus storing energy in the resonator. The rest of the circuit can be powered down in phase $\phi_{1}$ for reducing the operation power. At the end of this phase, the excitation source is switched off. During the subsequent phase $\phi_{2}$, the energy stored in the resonator starts to dissipate, generating a decaying sinusoidal ring-down current. If the driving frequency is perfectly matched to the resonance frequency, the initial amplitude of this current equals $V_{e x t} / R_{m}$ (where $V_{e x t}$ is the amplitude of the driving voltage). A lower amplitude is obtained if the driving frequency deviates from the resonance frequency. To convert this current into a voltage, the resonator is connected to an integrator that acts as a current-to-voltage converter. The high values of $R_{m}$ for the resonators we target is the main reason to choose an amplifier with a capacitive feedback instead of a resistive feedback, since the feedback resistor would have to be of the same order of magnitude as $R_{m}$, and would therefore consume substantial die area. A capacitor with an equivalent impedance consumes much less die area.

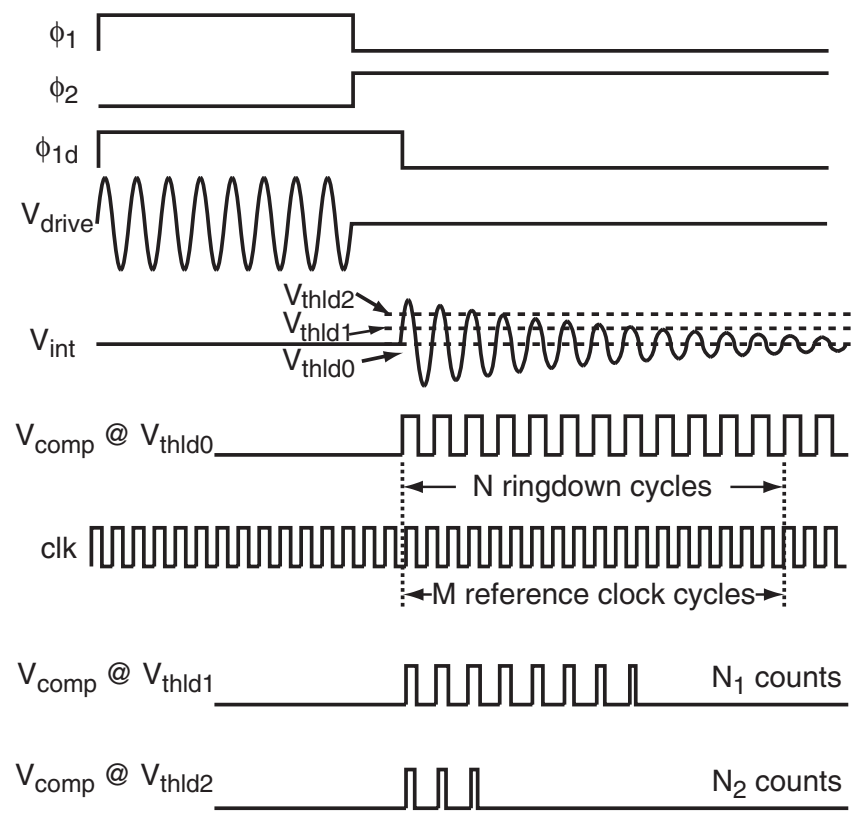

FIG. 3. Timing diagram of the readout, showing the control signals, the drive voltage $V_{\text {drive }}$, the output of the integrator $V_{\text {int }}$, the reference clock signal, and the output of the comparator $V_{\text {comp }}$ for resonance frequency measurement and quality factor measurement. 
To prevent charge stored on the parasitic shunt capacitor $C_{p}$ from being integrated, the integrator is briefly kept in unity-gain configuration at the beginning of phase $\phi_{2}$ by a delayed falling edge of phase $\phi_{1 \mathrm{~d}}$ (Fig. 3). As long as this delay is sufficiently long, the charge stored on $C_{p}$ is fully dissipated without causing an offset at the output of the integrator. This comes at the acceptable cost of loss in the initial part of the ring-down current. As a result, the operation of the circuit is independent of the parasitics, provided the operational transconductance amplifier (OTA) of the integrator has sufficient trans-conductance to ensure that the majority of the ring-down current flows into the integration capacitor, rather than into the parasitics. The integration of the ring-down current results a decaying sinusoidal ring-down voltage at the output of the integrator.

\section{B. Resonance frequency measurement}

To determine the resonance frequency $f_{\text {res }}$, the zerocrossings of the ring-down voltage $V_{\text {int }}$ are detected by the comparator (Fig. 2) while a zero-threshold level $V_{\text {thld }}$ is applied. The digital counter then counts the number of cycles $M$ of a reference clock with a clock frequency $f_{c l k}$ during a measurement time window consisting of a pre-defined number $N$ of ring-down cycles, as illustrated in Fig. 3. From this, the resonance frequency can be readily calculated as

$$
f_{\text {res }}=f_{c l k} \cdot \frac{N}{M} \text {. }
$$

However, Eq. (3) is only accurate when the pre-defined measurement time window contains an integer number of reference-clock cycles. Otherwise, it contains between $M$ and $(M+1)$ cycles, corresponding to a quantization error of at most one reference-clock cycle. This error can be reduced by increasing $f_{c l k}$.

This counting approach is preferred over the alternative of counting the number of ring-down cycles during a pre-defined number of reference-clock cycles, which would lead to much larger quantization errors ${ }^{17}$ due to the fact that the detectable number of ring-down cycles is limited by the relatively modest quality factor of our resonators to a few hundred.

To allow the comparator to detect as many zero-crossings as possible, it is important to ensure that the threshold of the comparator accurately matches with the steady-state value that the output of the integrator reaches at the end of the ring-down signal. However, several offset sources make this steady-state value hard to predict precisely. One offset source is related to the timing of the moment at which the current-tovoltage converter starts to integrate. Only when the unity-gain switch (phase $\phi_{1 \mathrm{~d}}$ ) of the integrator opens at the peak of the ring-down current, the output of the integrator begins to ring down at the steady state value, as a $90^{\circ}$ phase shift is added by the integration capacitance $C_{\text {int }}$. However, this cannot be guaranteed, as the timing of the peak of the ring-down current is not accurately known, because the exact resonance frequency is unknown. Moreover, an unknown phase shift is introduced due to the fact that the ring-down current divides between the shunt capacitor $C_{p}$ and the finite input impedance of the in-

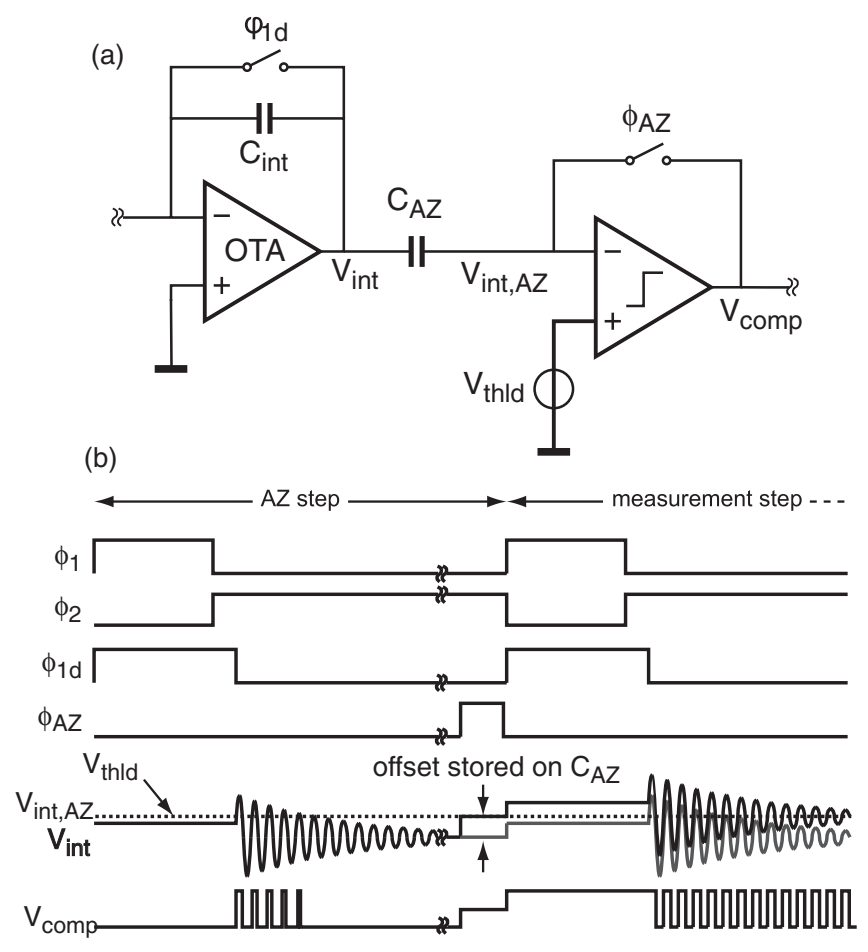

FIG. 4. (a) Circuit diagram of comparator auto-zeroing scheme, including an auto-zeroing capacitor $C_{A Z}$ and a feedback switch controlled by phase $\phi_{A Z}$. (b) Associated timing diagram, demonstrating the control signals of associated switches, the output signal of the integrator before auto-zeroing $\left(V_{\text {int }}\right)$ and after auto-zeroing $\left(V_{\text {int }, A Z}\right)$, and the output signal of the comparator $V_{\text {comp. }}$.

tegrator, which will also cause an offset at the output of the integrator.

To solve this offset issue, an auto-zeroing scheme is employed, as illustrated in Fig. 4. The resonator is excited twice. After the first excitation, the resonator is allowed to ring down completely. The comparator is then configured in unity-gain (phase $\phi_{\mathrm{AZ}}$ ), causing the difference between the steady-state output of the integrator and the input offset-voltage of the comparator to be stored on an auto-zeroing capacitor $\left(C_{A Z}\right)$. After the second excitation, the front-end circuit is operated as described before, and the comparator is operated as a regular comparator. Since $C_{A Z}$ still stores the same voltage difference, the level-shifted $V_{i n t, A Z}$ (Fig. 4) now has a steady-state value equal to the threshold of the comparator. Therefore, the comparator now precisely detects the zero-crossings, providing an accurate calculation of the resonance frequency.

\section{Quality factor measurement}

For quality factor measurements, two non-zero threshold levels $V_{\text {thld } 1}$ and $V_{\text {thld } 2}$ are applied. ${ }^{18}$ By counting the corresponding number of pulses, $N_{1}$ and $N_{2}$, that appear at the output of the comparator, an estimation of the exponentially decaying envelope can be made (Fig. 3). The quality factor can then be calculated as

$$
Q=\pi \cdot \frac{N_{1}-N_{2}}{\ln \left(V_{\text {thld } 2} / V_{t h l d 1}\right)} .
$$




\section{EXPERIMENTAL PROTOTYPE}

The prototype consists of a custom integrated circuit (IC) and a field-programmable gate array (FPGA). The custom IC contains the integrator, the auto-zeroed comparator with the associated switches and bias circuitry, as well as a voltage buffer that can be used to monitor the output of the integrator during measurements. The counter and digital circuits to generate the control signals for the switches have been implemented in the FPGA for flexibility, but could easily be cointegrated on the IC.

\section{A. Integrator design}

The integrator consists of an OTA with a programmable feedback capacitor. The OTA is designed as simple as possible, using a single common-source NMOS transistor $M_{1}$ (Fig. 5(a)). To allow a maximum output swing, a helper amplifier is used during phase $\phi_{1 \mathrm{~d}}$ to set the output voltage of the integrator to half of its supply ( $V_{\text {mid }}$ in Fig. 5). Instead of shorting the integration capacitor, this approach pre-charges it to an appropriate voltage to obtain a mid-supply reset level at the output. This helper amplifier is disconnected from the integrator and switched off during phase $\phi_{2}$ to save energy. Its implementation, shown in Fig. 5(b), consists of a pair of PMOS transistors, one of which effectively acts as a foldedcascode transistor in the feedback path of $M_{1}$. The feedback capacitance consists of three switchable capacitors in parallel, so that it can be digitally programmed between $0.15 \mathrm{pF}$ and $1.05 \mathrm{pF}$ to accommodate resonators with different mechanical resistances $R_{m}$.

The finite off-resistance of the switches connected to the virtual ground node of the integrator would give rise to a leakage current that is integrated and causes a droop on the output of the integrator, thus reducing the detection accuracy. To prevent this, nodes in the circuit that are potential sources of leakage to the virtual ground are bootstrapped to a replica $V_{r}$ of the virtual-ground voltage, which is generated using a diodeconnected NMOS transistor $M_{2}$ operating at the same overdrive voltage as the common-source NMOS transistor $M_{1}$ of the OTA, as illustrated in Fig. 5(c).

\section{B. Comparator design}

The comparator consists of a cascoded telescopic preamp and a common-source gain stage, as shown in Fig. 6. During auto-zeroing, the pre-amp is operated in unity-gain to store its input offset, in addition to the output offset voltage of the integrator, on the auto-zeroing capacitor $C_{A Z}$.

\section{FPGA and PCB implementation}

For flexibility, the control signals, as well as the implementation of the frequency counter, are generated off-chip using a FPGA board. A reference clock of $50 \mathrm{MHz}$ is applied on

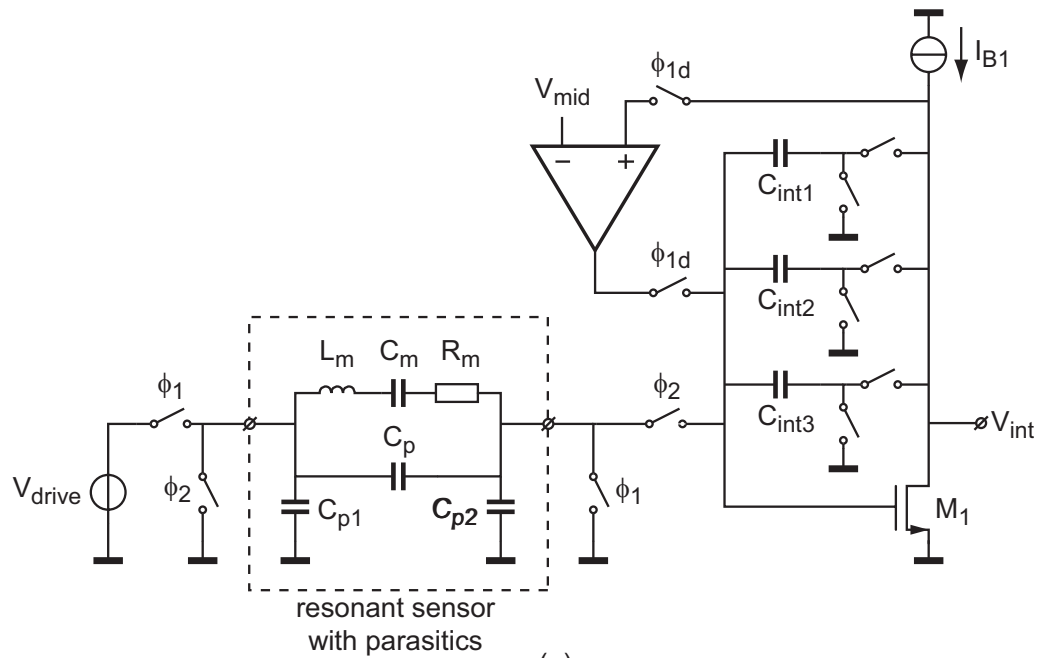

(a)
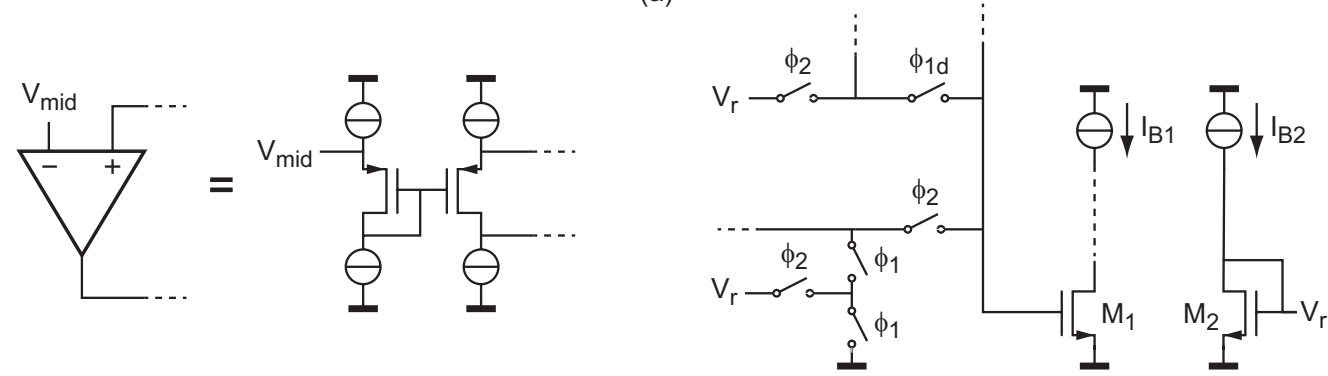

(b)

(c)

FIG. 5. (a) Circuit implementation of the integrator which consists of: a common-source NMOS transistor $M_{1}$ as amplifier, a helper amplifier to define output level of the integrator, and a switchable feedback capacitance. (b) Implementation of the helper amplifier. (c) Low-leakage implementation of the switches connected to the gate of $M_{1}$ by using a replica $V_{r}$ of the gate-source voltage of $M_{1}$, generated using an extra NMOS transistor $M_{2}$. 


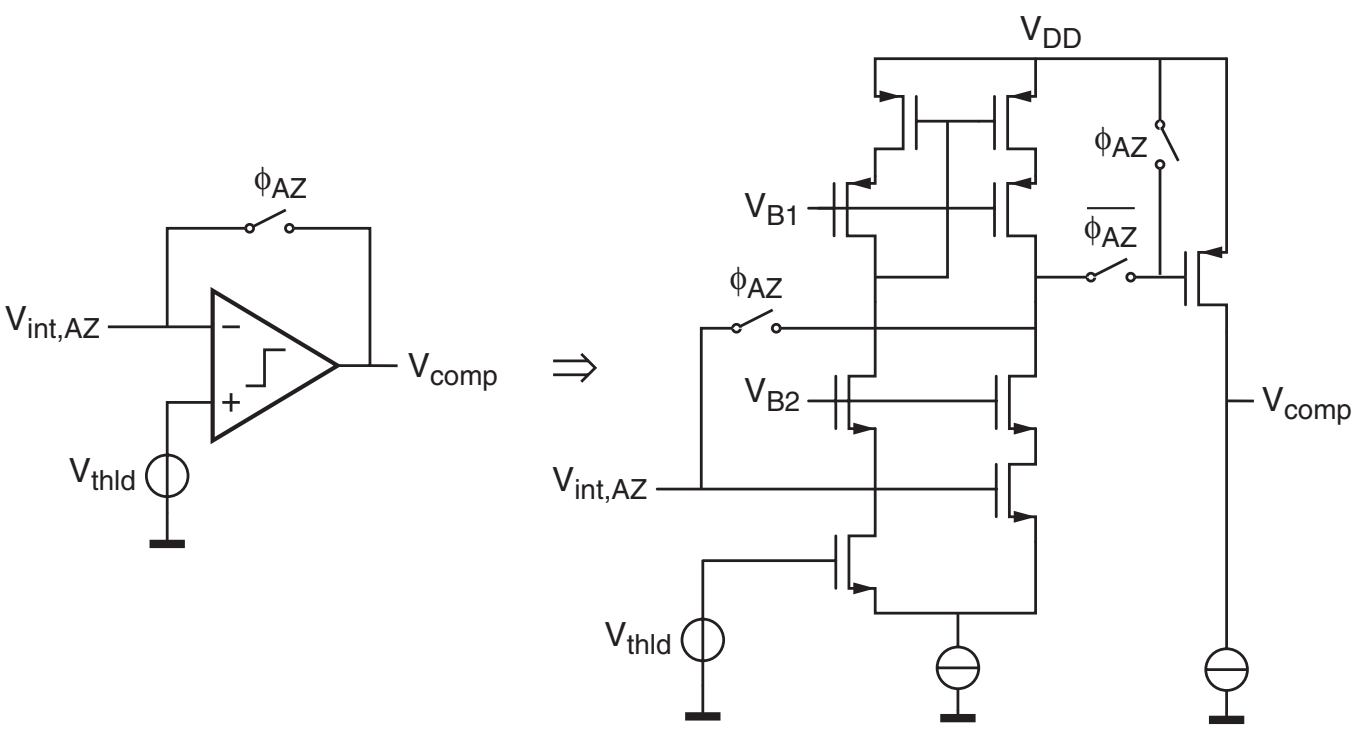

FIG. 6. Circuit implementation of the auto-zeroed comparator, which consists of a cascoded telescopic pre-amp and a common-course second stage.

board to accomplish the frequency counting. This reference clock is also used to generate all the control signals for the on-chip switches. A total measurement time of $1 \mathrm{~ms}$ is used for excitation and counting.

The FPGA also provides a square wave excitation signal which is filtered on a test PCB and applied to the resonator via switches integrated on chip. Moreover, adjustable threshold levels for the comparator are also produced on the PCB using resistive dividers which can be selected by using a programmable multiplexer.

\section{EXPERIMENTAL RESULTS}

A prototype of the readout circuit has been implemented in $0.35 \mu \mathrm{m}$ standard CMOS technology. A die photograph of the fabricated chip is shown in Fig. 7. This prototype was tested using a micro-machined clamped-clamped silicon nitride beam resonator with dimensions of $750 \mu \mathrm{m} \times 500 \mu \mathrm{m}$

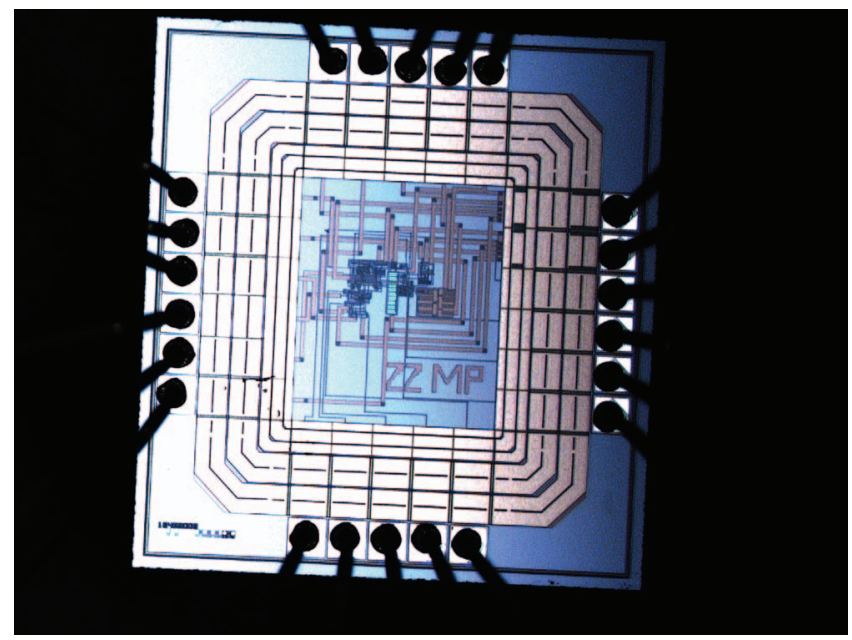

FIG. 7. Chip photograph of the readout circuit prototype. The chip is implemented in $0.35 \mu \mathrm{m}$ standard CMOS technology, with an area of $1.2 \mathrm{~mm}$ $\times 1.3 \mathrm{~mm}$. $\times 0.5 \mu \mathrm{m}$ (length $\times$ width $\times$ thickness), coated with poly(methyl methacrylate) polymer selected to be sensitive to ethanol. The micro-mechanical beam also consists of an integrated $400 \mathrm{~nm}$ thick aluminum nitride layer as the piezoelectric transducer covering a quarter of the device length that forms a parasitic parallel-plate capacitance of $3.2 \mathrm{pF}$. It is operated at a higher-order vibration mode with a resonance frequency of about $2 \mathrm{MHz}$ and a quality factor of about 670 at ambient conditions. An excitation signal at $2.006 \mathrm{MHz}$ with an amplitude of $200 \mathrm{mV}_{\mathrm{p}-\mathrm{p}}$ was applied. The resulting output signal of the integrator, observed using the on-chip voltage buffer, and the output signal of the comparator are obtained using an oscilloscope, as shown in Fig. 8. The integrator output is higher than mid-supply because the ring-down signal is level-shifted by the source-follower voltage buffer that prevents the integrator output from being loaded by the oscilloscope probe. The two subsequent excitations and ring-down steps, one for auto-zeroing and one for measurement, can be observed. To show the effects of auto-zeroing, measurements were taken without applying auto-zeroing by setting the autozeroing switch (phase $\phi_{A Z}$ in Fig. 4(a)) to remain open. As the results displayed in Fig. 8(b) show, the comparator detects much less ring-down cycles in this case.

For resonance frequency measurement, $N=150$ ringdown cycles are used. The results thus obtained have a quantization step of about $\delta f=500 \mathrm{~Hz}$, or $\delta f / f_{\text {res }}=250 \mathrm{ppm}$. Since the thermal noise is approximately at the same level, successive measurements randomly alternate between adjacent quantization levels, so that the uncertainty associated with a single measurement is about $\pm 250 \mathrm{ppm}$. The resolution can be further increased by averaging multiple measurements.

To determine the quality factor, threshold-levels of $0.1 \mathrm{~V}$ and $0.2 \mathrm{~V}$ were applied to the comparator. The number of cycles obtained for each threshold at the output of the comparator was counted (Fig. 9). A quality factor of $Q=$ 661 was found by calculation using Eq. (4), which is in good agreement with the quality factor of $Q=667$ obtained using impedance analysis. 
(a)

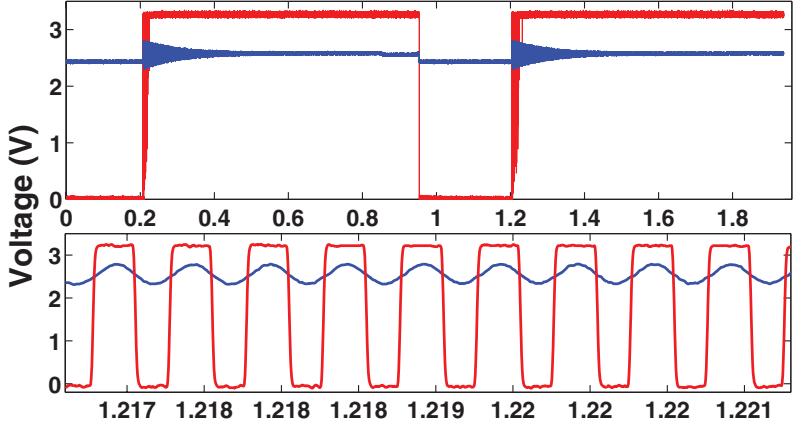

(b)

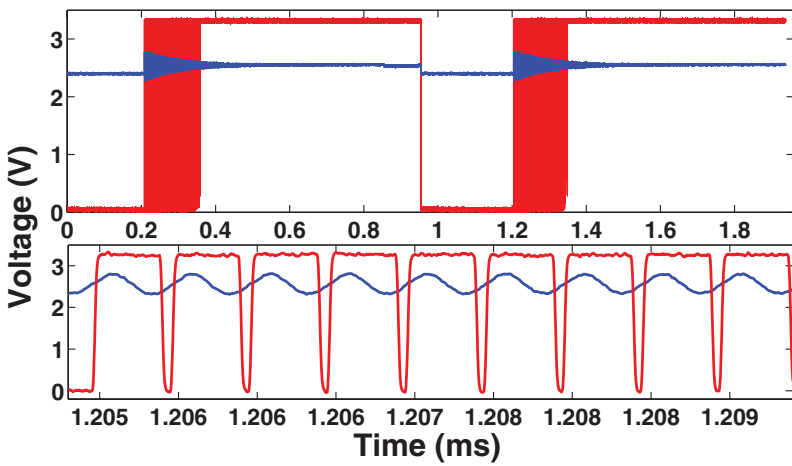

FIG. 8. Transient measurements obtained using an oscilloscope: buffered integrator output (decaying sinusoidal wave); comparator output (square wave); and zoom-in of the signals (a) with auto-zeroing and (b) without auto-zeroing.

Figure 10 shows the measured resonance frequency shift and relative change of the resonance frequency as a function of the dc bias voltage applied across the resonator. This frequency shift is due to a stress formation within the length restricted beam. ${ }^{2}$ The bias dependency of $0.035 \% / \mathrm{V}$ found using the ring-down circuit agrees well with the results obtained using an impedance analysis $(0.03 \% / \mathrm{V})$.

Figure 11 shows the transient response of the resonator in a dry nitrogen flow during exposure to ethanol at $1000 \mathrm{ppm}$
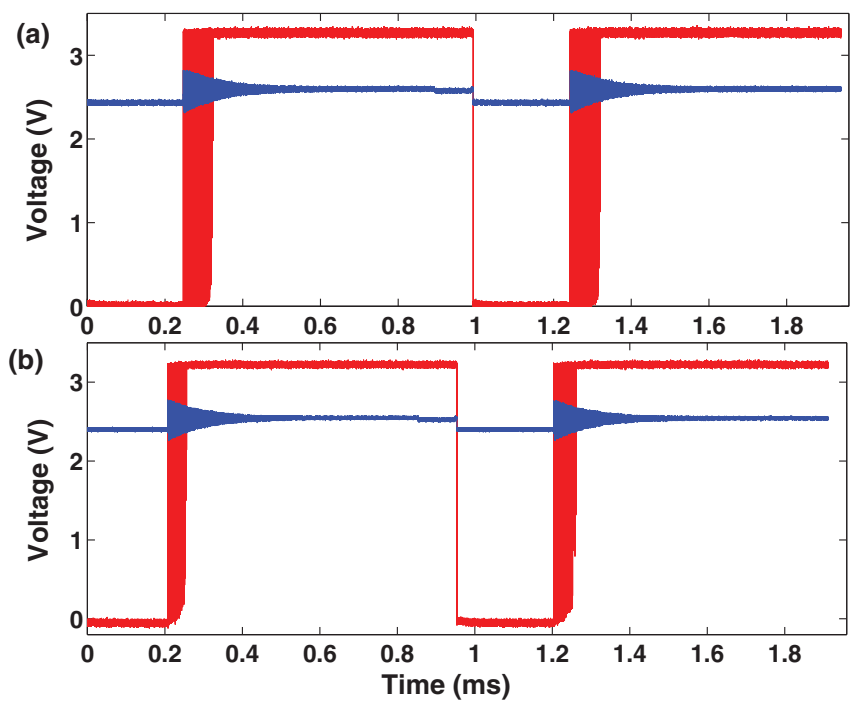

FIG. 9. Quality factor measurement results (decaying sinusoidal wave at the buffered output of the integrator and square wave at the output of the comparator), when a threshold-level of (a) $0.1 \mathrm{~V}$ and (b) $0.2 \mathrm{~V}$ was applied.

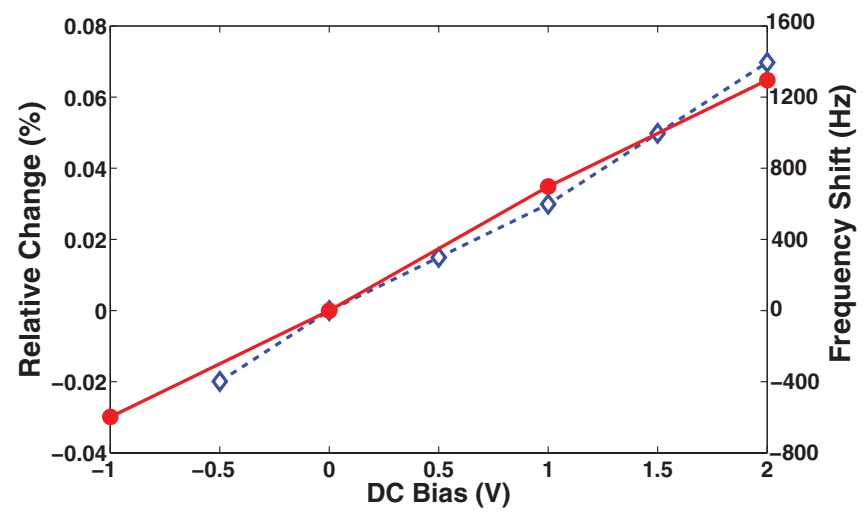

FIG. 10. Measured resonance frequency shift and its relative change, with respect to the resonance frequency with $0 \mathrm{~V}$ across the resonator, as a function of dc bias voltage using the presented interface (dashed line with diamond marker) and impedance analysis (solid line with circle marker).

concentration. For each data point of this transient response, an average resonance frequency of 400 measurements was taken to reduce the measurement uncertainty, by $\sim 20$ times, to $\pm 12.5 \mathrm{ppm}$. This leads to a measurement time of about $1 \mathrm{~s}$. The modal frequency measured as $2 \mathrm{MHz}$ shifted to $2.12 \mathrm{MHz}$ in dry nitrogen flows as a result of known sensitivity of the coating to humidity. When the resonator is exposed to the ethanol vapor, the modal frequency is decreasing due to the absorption of the volatile compound in the polymer coating, as previously described in detail. ${ }^{2}$ The ethanol flow was applied twice, and the resonator was exposed to dry air after each exposure to demonstrate the recovery.

The circuit implemented on chip consumes $36 \mu \mathrm{A}$ from a $3.3 \mathrm{~V}$ supply. This corresponds to about $120 \mathrm{~nJ}$ per measurement step of $1 \mathrm{~ms}$. This can be further reduced by optimizing the circuit and reducing the measurement time. Even though this number does not include the excitation and counting circuitry, the energy consumed by these components is not expected to be dominant in the final system.

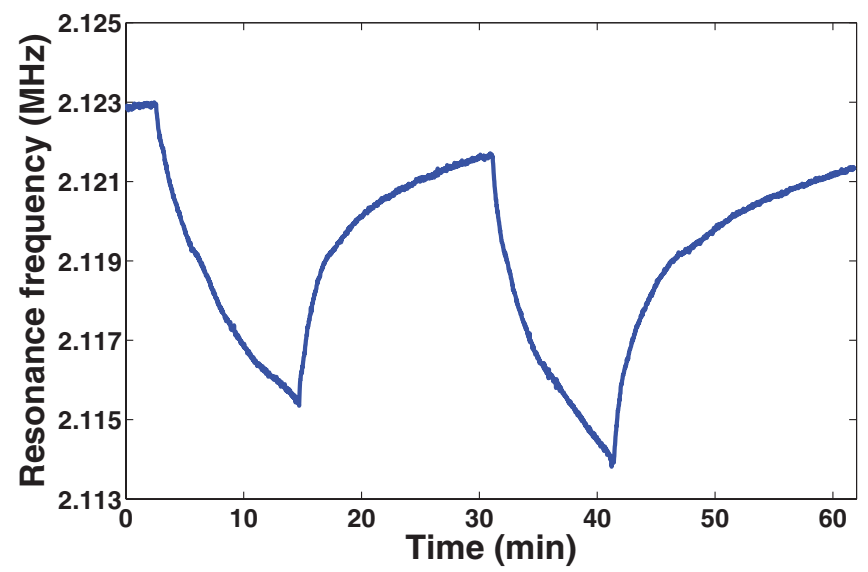

FIG. 11. Transient response of the resonator to ethanol exposure using the presented readout circuit. The modal frequency decreases during ethanol exposure due to the absorption of the volatile compound in the polymer coating of the resonator. 


\section{CONCLUSIONS}

A readout circuit for micro-mechanical resonant sensors has been presented here. In contrast with oscillator-based interfaces, the presented readout circuit can handle resonators with a large parasitic capacitance, and can readily be used to extract both the resonance frequency and quality factor using counting techniques while consuming significantly less energy. A low-energy integrated prototype of the readout circuit was realized in $0.35 \mu \mathrm{m} \mathrm{CMOS} \mathrm{technology.} \mathrm{The} \mathrm{readout}$ circuit was demonstrated to be able to detect the frequency of a $2 \mathrm{MHz}$ resonator with $500 \mathrm{~Hz}$ resolution for a single ring-down measurement, within a $1 \mathrm{~ms}$ long measurement at an energy consumption of $120 \mathrm{~nJ}$. There clearly exists a trade-off between improving the accuracy of the readout circuit by averaging multiple measurements and reducing energy consumption.

Furthermore, quality factor and dc sensitivity measurements obtained using this chip, in combination with a MEMS resonator, are in good agreement with results from bench-top electronics. Finally, the chemical detection potential of the system has been demonstrated with a sensor element coated with a polymer functionalization layer, during an exposure to ethanol. The sensing system can detect low-ppm concentrations of vapors. The low energy consumption of the readout circuit makes ring-down-based interfacing a promising approach for readout of resonant sensors in energy-constrained applications.

\section{ACKNOWLEDGMENTS}

The authors thank Ms. Yvonne van Andel for fabrication of resonators, $\mathrm{Mr}$. Zu-yao Chang for his help in PCB design and measurements, and Ling Sieben-Xu for assistance in device characterization setup.

${ }^{1}$ P. S. Waggoner and H. G. Craighead, Lab Chip 7, 1238-1255 (2007).

${ }^{2}$ D. M. Karabacak, S. H. Brongersma, and M. Crego-Calama, Lab Chip 10, 1976-1982 (2010).

${ }^{3}$ D. M. Karabacak, V. Yakhot, and K. L. Ekinci, Phys. Rev. Lett. 98, 254505 (2007)

${ }^{4}$ S. C. Masmanidis, R. B. Karabalin, I. De Vlaminck, G. Borghs, M. R. Freeman, and M. L. Roukes, Science 317, 780-783 (2007).

${ }^{5}$ R. B. Karabalin, M. H. Matheny, X. L. Feng, E. Defaÿ, G. Le Rhun, C. Marcoux, S. Hentz, P. Andreucci, and M. L. Roukes, Appl. Phys. Lett. 95, 103111 (2009).

${ }^{6}$ K. L. Ekinci, Small 1, 786-797 (2005).

${ }^{7}$ M. Rodahl, F. Höök, A. Krozer, P. Brzezinski, and B. Kasemo, Rev. Sci. Instrum. 66, 3924-3930 (1995).

${ }^{8}$ M. Rodahl and B. Kasemo, Rev. Sci. Instrum. 67, 3238-3241 (1996).

${ }^{9}$ M. A. P. Pertijs, Z. Zeng, D. M. Karabacak, M. Crego-Calama, and S. H. Brongersma, "An energy-efficient interface for resonant sensors based on ring-down measurement," 2012 IEEE International Symposium on Circuits and Systems (ISCAS), 20-23 May 2012, pp. 990-993.

${ }^{10}$ H. A. C. Tilmans, J. Micromech. Microeng. 7, 285-309 (1997).

${ }^{11}$ K. E. Wojciechowski, Ph.D. Dissertation, University of California, Berkeley, 2005.

${ }^{12}$ A. O. Niedermayer, E. K. Reichel, and B. Jakoby, Sens. Actuators, A 156, 245-250 (2009).

${ }^{13}$ J. Verd, A. Uranga, G. Abadal, J. L. Teva, F. Torres, J. L. Lopez, E. PerezMurano, J. Esteve, and N. Barniol, IEEE Electron Device Lett. 29, 146-148 (2008).

${ }^{14}$ C. Hagleitner, D. Lange, A. Hierlemann, O. Brand, and H. Baltes, IEEE J. Solid-State Circuits 37, 1867-1878 (2002).

${ }^{15}$ V. Petrescu, J. Pettine, D. M. Karabacak, M. Vandecasteele, M. C. Calama, and C. Van Hoof, "Power-efficient readout circuit for miniaturized electronic nose," 2012 IEEE International Solid-State Circuits Conference Digest of Technical Papers (ISSCC), 19-23 February 2012, pp. 318-320.

${ }^{16}$ J. Pettine, M. Patrascu, D. M. Karabacak, M. Vandecasteele, V. Petrescu, S. H. Brongersma, M. Crego-Calama, and C. Van Hoof, Sens. Actuators, A 189, 496-503 (2013).

${ }^{17}$ K. Zeng, K. G. Ong, C. Mungle, and C. A. Grimes, Rev. Sci. Instrum. 73, 4375-4380 (2002)

${ }^{18}$ K. Zeng and C. A. Grimes, Rev. Sci. Instrum. 75, 5257-5261 (2004). 